\title{
THE COHOMOLOGY RINGS OF CERTAIN FINITE PERMUTATION REPRESENTATIONS ${ }^{1}$
}

\author{
JAMES V. BLOWERS
}

\begin{abstract}
In this paper the concept of join of two permutation representations is defined and the cohomology of this join is computed and shown to have trivial cup-products. This computation is then used to compute the cohomology groups of the $p$-Sylow subgroup of a symmetric group of order $n$ acting on the set of $n$ elements, and it is shown that the ring structure on these groups is not finitely generated, although it is transitive.
\end{abstract}

I. Introduction. Let $(G, X)$ and $(L, Y)$ be permutation representations; i.e., $G$ is a group acting on the set $X$ and $L$ is a group acting on the set $Y$. The ir join, denoted $(G, X) *(L, Y)$, is defined to be the permutation representation $(G \times L, X \amalg Y)$, where the action is given by $(g, l) x=g x$; $(g, l) y=l y$. In this note we shall exhibit a projective resolution for $(G, X) *$ $(L, Y)$ and the result will be used to compute a projective resolution for ( $G \int L, X \times Y$ ) where $G \int L$ is the "left-handed" wreath product defined in Evens [3, p. 54] and $G \int L$ acts in the usual fashion on $X \times Y$. This will allow us to compute the cohomology of the join and wreath products, and enough of the structure of the cohomology rings will be computed to show (1) the cohomology ring of $(G, X) *(L, Y)$ is trivial, and hence not finitely generated; $(2)$ the cohomology ring of the $p$-Sylow subgroup of the symmetric group of order $p^{r}$ acting on the set of $p^{r}$ elements is not finitely generated, even though the representation is transitive. See Snapper [6] and Harris [4] for details on cohomology of permutation representations.

Presented to the Society, August 23, 1972; received by the editors September 18, 1973.

AMS (MOS) subject classifications (1970). Primary 20J05, 18H10; Secondary 20B05, 18G10, 20D20, 18G25, 18G40, 20F25.

Key words and phrases. Join of permutation representations, projective resolution, suspended chain complex, wreath product, spectral sequence, cup-products, $p$-Sylow subgroup, symmetric group, transitive permutation representation, finite generation, cohomology of permutation representations.

1 This paper is based on a dissertation presented to the faculty of Nor thwestern University in partial fulfillment of the requirements of the degree of Doctor of Philosophy, June 1972. The work was supported in part by National Defense Education Act Title IV Fellowship No. 68-01201. 
II. Joins. Let $F$ be a field. For any augmented chain complex $K$ over $F$, define the suspended chain complex, $s K$, to be the complex obtained by raising dimensions by one and using $F$ and the augmentation for the zerodimensional module and boundary. Let $C$ and $D$ be augmented chain complexes. Define the join of $C$ and $D$, denoted $C * D$, by $s(C * D)=s C * s D$. We then have the following proposition:

Proposition 1. Let $(G, X)$ and $(L, Y)$ be permutation representations. If $C$ is a $(G, X)$-projective resolution of $F$; i.e., $C$ is $\mathcal{F}_{\text {-projective (Harris }}$ $[4$, p. 271]) and $C$ is $(G, X)$-exact (see Snapper [7, p. 1052]), and D is an $(L, Y)$-projective resolution of $F$, then $C * D$ is a $(G, X) *(L, Y)$-projective resolution of $F$.

Proof. The stabilizer set (see Harris $[4$, p. 272] for definition) of $(G, X)$ $*(L, Y)$ is given by

$$
\overline{\mathscr{L}}_{*}=\left\{G \times K, H \times L, H \times K \mid H \in \mathcal{F}_{(G, X)}, K \in \mathscr{F}_{(L, Y)}\right\} .
$$

We have that $C * D \cong C \otimes D \oplus C \otimes F \oplus F \otimes D$, as $G \times L$-modules. In Eilenberg-Moore $[1$, p. 27], we find that for any permutation representation $(M, Z)$, the $(M, Z)$-projective modules are $M$-direct summands of direct sums of modules of the form $F M \otimes_{F N} F$, where $N$ ranges over $\mathcal{F}_{(M, Z)^{*}}$. From this definition it is straightforward to prove that $C \otimes D, C \otimes F$, and $F \otimes D$ are $(G, X) *(L, Y)$-projective.

To show $C * D$ is $(G, X) *(L, Y)$-exact, we have to show that for any $H \in \mathscr{F}_{*}$ there is a contracting chain homotopy on $C * D$ that commutes with $H$. Without loss of generality we can assume that $H=M \times K$, where $M=G$ or $M \in \mathcal{F}_{(G, X)}$, and $K \in \mathcal{F}_{(L, Y)}$. Let $s$ be a $K$-homotopy for $D$. Let $s^{\prime}$ be the corresponding $K$-homotopy for $s D$. Then $1 \otimes s^{\prime}$ is an $M \times K$-homotopy for $s C \otimes s D=s(C * D)$. Lowering dimensions by one, we obtain an $M \times K-$, and hence an $H$-homotopy for $C * D$. Hence $C * D$ is a $(G, X) *(L, Y)$-complex.

Let $(G, X)$ and $(L, Y)$ be permutation representations and consider $\left(G \int L, X \times Y\right)$. Note that $G \int L$ is the semidirect product of $G$ and $L^{n}$, where $n$ = number of elements in $X$, and that $\left(L^{n}, X \times Y\right)$ is the $n$-fold join of $(L, Y)$. This leads to the following:

Proposition 2. Let $(G, X)$ and $(L, Y)$ be permutation representations with $G$ acting freely on $X=\{1, \cdots, n\}$. Then if $W$ is a $G$-projective resolution of $F$ and $C$ is an $(L, Y)$-projective resolution of $F$, and $J^{n} C$ denotes $C * \ldots * C$ ( $n$ times), then $W \otimes J^{n} C$ is a $\left(G \int L, X \times Y\right)$-projective resolution of. $F$, where the action is given by 


$$
g\left(l_{1}, \ldots, l_{n}\right)\left(w \otimes c_{1} * \ldots * c_{n}\right)=\epsilon g w \otimes\left(l_{g}^{-1} c_{g^{-1}(1)} * \cdots * l_{g^{-1}(n) g^{-1}(n)} c^{-1}\right),
$$

where $\epsilon$ is a certain sign that depends on permuting odd-dimensional (in the suspended sense) chains past each other.

Proof. This is a straightforward generalization of Evens [2, p. 231].

This resolution leads to the following generalization of Nakaoka's theorem [5, Theorem 3.3, p. 237]:

Theorem 1. If $G$ acts freely on $X$ and $L$ acts on $Y$, then we have

$$
H^{*}\left(\left(G \int L, X \times Y\right), F\right) \cong H^{*}\left(G, H^{*}\left(J^{n}(L, Y), F\right)\right)
$$

as additive groups, where $J^{n}(L, Y)$ denotes the $n$-fold join of $(L, Y)$.

Proof. By Proposition 2, if $W$ is a $G$-projective resolution of $F$ and $C$ is an $(L, Y)$-projective resolution of $F$, then $W \otimes J^{n} C$ is a $\left(G \int L, X \times Y\right)$ projective resolution of $F$. Hence, if $N$ denotes $L^{n}$,

$$
\begin{aligned}
H^{*}\left(\left(G \int L, X \times Y\right), F\right) & \cong H^{*}\left(\operatorname{Hom}_{G \int L}\left(W \otimes J^{n} C, F\right)\right) \\
& \cong H^{*}\left(\operatorname{Hom}_{G}\left(W, \operatorname{Hom}_{N}\left(J^{n} C, F\right)\right)\right) .
\end{aligned}
$$

There is a monomorphism of degree one $i_{c}: J^{n} C \rightarrow s J^{n} C=(s C)^{n}$. This induces a map of double complexes

$$
i_{c}^{*}: \operatorname{Hom}_{G}\left(W, \operatorname{Hom}_{N}\left(J^{n} C, F\right)\right) \rightarrow \operatorname{Hom}_{G}\left(W, \operatorname{Hom}_{N}\left((s C)^{n}, F\right)\right) .
$$

Each side is a double complex, so that $i_{c}^{*}$ induces a map

(1) $\quad i_{c}^{*}: H^{*}\left((G, X), H^{*}\left(J^{n}(L, Y), F\right)\right) \rightarrow H^{*}\left((G, X), s H^{*}\left(J^{n}(L, Y), F\right)\right)$,

i.e., $i_{c}^{*}$ now is a map from the $E_{2}$ term of the second spectral sequence of the left-hand side to the $E_{2}$ term of the second spectral sequence of the right-hand side. There is an obvious "splitting"

$$
r:(G, X) \rightarrow\left(G \int L, X \times Y\right), \quad j:\left(G \int L, X \times Y\right) \rightarrow(G, X)
$$

which induces a similar splitting on the cohomology level. Thus $j^{*}$, which is the inflation map from $(G, X)$ to $\left(G \int L, X \times Y\right)$ is a monomorphism. The spectral sequence of the left-hand side of $(1)$ is the spectral sequence of Snapper [6, Theorem 19.1, p. 158] of the morphism $j$; hence $j^{*}$ is the edge homomorphism on the base, and since $j^{*}$ is a monomorphism, the base is never hit by any of the differentials of the spectral sequence. The map $i_{c}^{*}$ is an isomorphism above the base and is the zero map on the base of $E_{2}$ and 
hence of $E_{\infty^{\prime}}$. Since the coefficients are in a field, we conclude that $i_{c}^{*}$ on the final term is an epimorphism with kernel

$$
H^{*}\left(G, H^{0}\left(J^{n}(L, Y), F\right)\right)=H^{*}(G, F)
$$

Similar to Nakaoka [5, p. 237], we have a cochain equivalence $\psi: s C^{*}$ $\rightarrow s H^{*}$ which induces a cochain equivalence

$$
\bar{\psi}: \operatorname{Hom}_{G}\left(W, \operatorname{Hom}_{N}((s C), F)\right) \rightarrow \operatorname{Hom}_{G}\left(W,\left(s H^{*}\right)^{n}\right),
$$

where $s C^{*}=\operatorname{Hom}_{L}(s C, F)$, and $s H^{*}=\operatorname{Hom}_{L}(s H, F)$. Since the left-hand side of (2) is equal to $\operatorname{Hom}_{G}\left(W,\left(s C^{*}\right)^{n}\right)$, this yields an isomorphism of additive groups:

$$
\bar{\psi}^{*}: H^{*}\left(\operatorname{Hom}_{G}\left(W,\left(s C^{*}\right)^{n}\right)\right) \rightarrow H^{*}\left((G, X),\left(s H^{*}\right)^{n}\right)
$$

The right-hand side can be shown to be the same as $H^{*}\left((G, X), \tilde{H}^{*}\left(J^{n}(L, Y), F\right)\right)$ where $\widetilde{H}^{*}$ denotes reduced cohomology: $\widetilde{H}^{0}=0$ and $\widetilde{H}^{n}=H^{n}$ for $n>0$. If we combine $i_{c}^{*}$ with $\bar{\psi}^{*}$, we obtain an epimorphism

$$
\phi: H^{*}\left(\left(G \int L, X \times Y\right), F\right) \rightarrow H^{*}\left(G, \widetilde{H}\left(J^{n}(L, Y), F\right)\right)
$$

(since $G$ acts freely on $X, H^{*}((G, X), A)=H^{*}(G, A)$ for any $G$-module $A$ ) with kernel $H^{*}\left(G, H^{0}\left(J^{n}(L, Y), F\right)\right)$. Thus

$$
H^{*}\left(\left(G \int L, X \times Y\right), F\right) \cong \operatorname{Ker} \phi \oplus \operatorname{Im} \phi=H^{*}\left(G, H^{*}\left(J^{n}(L, Y), F\right)\right) \text {. }
$$

III. Products. The product on the join of two permutations is given by the following:

Theorem 2. If $(G, X)$ and $(L, Y)$ are permutation representations, and if $\alpha, \beta \in H^{*}((G, X) *(L, Y), F)$ with $\operatorname{deg} \alpha>0, \operatorname{deg} \beta>0$, then $\alpha \cup \beta=0$.

Proof. Let $C$ be a $(G, X)$-projective resolution of $F$ and $D$ be an $(L, Y)$ projective resolution of $F$. Then a diagonal map $\Delta$ for $(G, X) *(L, Y)$ is given by the following. Let $\delta^{C}: C \rightarrow C \otimes C$ and $\delta^{D}: D \rightarrow D \otimes D$ be the diagonal maps for $C$ and $D$ respectively. Further, let

$$
\Psi:(s C \otimes s C) \otimes(s D \otimes s D) \rightarrow(s C \otimes s D) \otimes(s C \otimes s D)
$$

be the map which shuffles factors with a sign determined by the suspended degrees, and let $1 \in F$ denote the unit of $s C_{0}$ and $s D_{0}$. We can consider $\delta^{C}$ and $\delta^{D}$ to map into $s C \otimes s C$ and $s D \otimes s D$ respectively. Then $\Delta$ is given by 


$$
\begin{aligned}
& \Delta_{p, q}\left(c_{m} * d_{n}\right)=\Psi\left(\delta{ }_{p, m-p}^{C} c_{m} \otimes\left(1 \otimes d_{m}\right)\right) \quad \text { if } p \leq m, \\
& =\Psi\left(\left(c_{n} \otimes 1\right) \otimes \delta_{p-m-1, q}^{D} d_{n}\right) \quad \text { if } p>m .
\end{aligned}
$$

The computational proof that $\Delta$ is a diagonal map is omitted for the sake of brevity; instead, it shall be shown where $\Delta$ came from: Let $C$ and $D$ be the standard complexes for $(G, X)$ and $(L, Y)$ (see Snapper [6, p. 135]), and let $E$ be the standard complex for $(G, X) *(L, Y)$. Then $C * D \rightarrow E$ by

$$
\left(x_{1}, \ldots, x_{m}\right) *\left(y_{1}, \ldots, y_{n}\right) \rightarrow\left(x_{1}, \ldots, x_{m}, y_{1}, \ldots, y_{n}\right)
$$

and if $\Delta: C * D \rightarrow(C * D) \otimes(C * D)$ is defined as above and $\Delta^{\prime}: E \rightarrow E \otimes E$ is defined in the usual fashion for a standard complex, then the following diagram commutes:

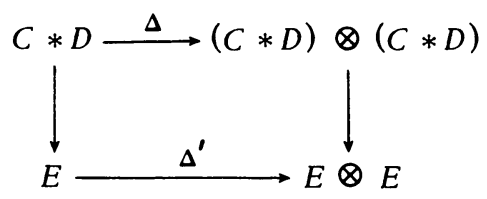

Observation of the formula (3) for $\Delta$ reveals that application of $\Delta$ always results in an element of $(C * F) \otimes(C * D)$ or $(C * D) \otimes(F * D)$, i.e., one of the two factors is always on the "edge". Hence if any cocycle $f \epsilon$ Hom $_{G \times L}(C * D, F)$ can be changed by a coboundary so that $f$ is zero on either edge, then products of positive-dimensional cohomology classes will always be zero. To show this can be done, note that $\operatorname{Hom}_{G}(C * D, F)$ has trivial cohomology (a $G$-contracting homotopy is given by $1 * s$, where $s$ is a contracting homotopy for $D$ ) and that

$$
0 \rightarrow \operatorname{Hom}_{G \times L}(C * D, F) \rightarrow \operatorname{Hom}_{G}(C * D, F) .
$$

splits over $F$ with $\operatorname{Hom}_{G \times L}(C * F, F)=\operatorname{Hom}_{G}(C * F, F)$, since $L$ acts trivially on $F$. Let $f=\delta h$, where $h \in \operatorname{Hom}_{G}(C * D, F)$. Then $b=b^{\prime}+b^{\prime \prime}$, where $b^{\prime} \in \operatorname{Hom}_{G \times L}(C * D, F)$, and $b^{\prime \prime}$. is in the complementary summand of $\operatorname{Hom}_{G \times L}(C * D, F)$ in $\operatorname{Hom}_{G}(C * D, F)$. Then since $b^{\prime \prime}=0$ on $C * F$, we have that $f$ on $C * F$ is the same as $\delta b$ on $C * F$, hence $f-\delta b$ is zero on the edge $C * F$, as required. This completes the proof.

Corollary. If $G$ acts freely on $X$ and $L$ acts freely on $Y$, then $H^{*}((G, X) *(L, Y), F)$ is not finitely generated.

Proof. A theorem of Evens [2, Corollary 7.2, p. 237] states that both $H^{*}(G, F)$ and $H^{*}(H, F)$ have an infinite number of additive generators. Since 
products are zero in $H^{*}((G, X) *(L, Y), F)$, it follows that $H^{*}((G, X) *(L, Y), F)$ has an infinite number of ring generators. Hence, $H^{*}((G, X) *(L, Y), F)$ is not finitely generated.

That even for transitive $(G, X)$ the ring $H^{*}((G, X), F)$ need not be $f$ nitely generated is shown by:

Theorem 3. The cohomology ring of the p-Sylow subgroup of the symmetric group on $p^{t}$ elements acting on the set of $p^{t}$ elements is not finitely generated for $t \geq 2$.

Proof. It is well known that if $(G, X)$ denotes the permutation representation of the theorem and if $P$ denotes the group $Z_{p}$, then

$$
(G, X)=\left(P \int L, P \times Y\right),
$$

where $(L, Y)$ is the $p$-Sylow subgroup of the symmetric group on $p^{t-1}$ elements acting on the set of $p^{t-1}$ elements. The permutation representation $\left(L^{p}, X\right)$ is $J^{p}(L, Y)$, so that $H^{*}\left(\left(L^{p}, X\right), F\right)$ has an inf inite number of additive generators, by an induction argument. One can also show that $H^{*}\left(\left(L^{p}, X\right), F\right)^{P}$ also has an infinite number of additive generators, where $A^{P}$ denotes the $P$-fixed elements of $A$. Further, there is a restriction map:

$$
\text { res: } H^{*}((G, X), F) \rightarrow H^{*}((H, X), F)
$$

(see Snapper [6, p. 145]) which maps into $H^{*}((H, X), F)^{P}$. This restriction is induced by the map $\epsilon \otimes \mathrm{id}: J^{p} C \rightarrow W \otimes J^{p} C$. The following diagram commutes, where $N$ now denotes $L^{p}$ :

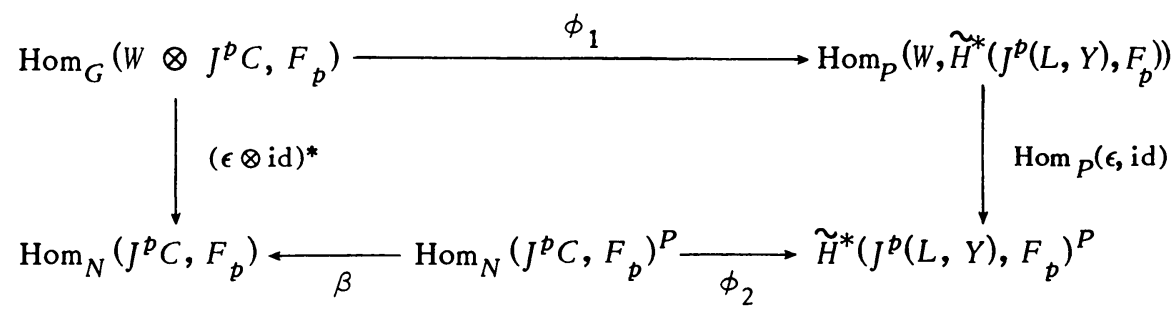

The map $\phi_{1}$ is the isomorphism of Theorem 1 , and $\phi_{2}$ is the same isomorphism without $W$. The map $\beta$ is an inclusion map. In cohomology, $\operatorname{Im}(\epsilon \otimes \mathrm{id})^{*}$ $C \operatorname{Im} \beta$ and $\phi_{1}$ and $\phi_{2}$ become epimorphisms which are isomorphisms above the base of the spectral sequence of Theorem 1. So above the base res carries over to projection onto the zeroth cohomology group. One can work out separately that on the base res also carries over to projection onto the zeroth cohomology group. Hence res is a projection, hence onto. 
Since res preserves ring structure, we conclude $H^{*}\left((G, X), F_{p}\right)$ has a homomorphic image which is not finitely generated; hence $H^{*}\left((G, X), F_{p}\right)$ is not finitely generated either.

\section{BIBLIOGRA PHY}

1. Samuel Eilenberg and J. C. Moore, Foundations of relative homological algebra, Mem. Amer. Math. Soc. No. 55 (1965). MR 31 \#2294.

2. Leonard Evens, The cohomology ring of a finite group, Trans. Amer. Math. Soc. 101 (1961), 224-239. MR 25\#1191.

3. - A generalization of the transfer map in the cohomology of groups, Trans. Amer. Math. Soc. 108 (1963), 54-65. MR 27 \#3686.

4. Morton E. Harris, Generalized group cohomology, Fund. Math. 65 (1969), 269-288. MR 40 \#5701.

5. Minoru Nakaoka, Homology of the infinite symmetric group, Ann. of Math. (2) 73 (1961), 229-257. MR 24 \#A1721.

6. Ernst Snapper, Cohomology of permutation representations. I. Spectral sequences, J. Math. Mech. 13 (1964), 133-161. MR 28 \#1226.

7. - Cohomology of permutation representations. II. Cup product, J. Math. Mech. 13 (1964), 1047-1064.

DEPARTMENT OF MATHEMATICS, NORTHWESTERN UNIVERSITY, EVANSTON, ILLINOIS 60201

Current address: AFATL-DLYA, Eglin AFB, Florida 32542 